\title{
Front Matter: Volume 9750
}

, "Front Matter: Volume 9750," Proc. SPIE 9750, Integrated Optics: Devices, Materials, and Technologies XX, 975001 (21 July 2016); doi:

$10.1117 / 12.2239576$

SPIE. Event: SPIE OPTO, 2016, San Francisco, California, United States 


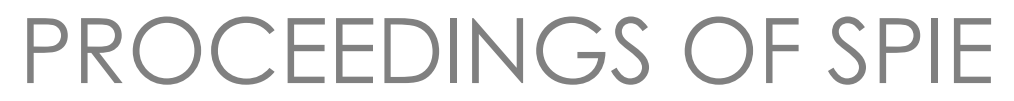

\title{
Integrated Optics: Devices, Materials, and Technologies XX
}

\author{
Jean-Emmanuel Broquin \\ Gualtiero Nunzi Conti \\ Editors
}

\author{
15-17 February 2016 \\ Sponsored and Published by \\ SPIE
}

San Francisco, California, United States

Volume 9750 
The papers in this volume were part of the technical conference cited on the cover and title page. Papers were selected and subject to review by the editors and conference program committee. Some conference presentations may not be available for publication. Additional papers and presentation recordings may be available online in the SPIE Digital Library at SPIE Digitallibrary.org.

The papers reflect the work and thoughts of the authors and are published herein as submitted. The publisher is not responsible for the validity of the information or for any outcomes resulting from reliance thereon.

Please use the following format to cite material from these proceedings:

Author(s), "Title of Paper," in Integrated Optics: Devices, Materials, and Technologies XX, edited by Jean-Emmanuel Broquin, Gualtiero Nunzi Conti, Proceedings of SPIE Vol. 9750 (SPIE, Bellingham, WA, 2016) Article CID Number.

ISSN: 0277-786X

ISSN: 1996-756X (electronic)

ISBN: 9781628419856

Published by

SPIE

P.O. Box 10, Bellingham, Washington 98227-0010 USA

Telephone +1 3606763290 (Pacific Time) · Fax + 13606471445

SPIE.org

Copyright (C) 2016, Society of Photo-Optical Instrumentation Engineers.

Copying of material in this book for internal or personal use, or for the internal or personal use of specific clients, beyond the fair use provisions granted by the U.S. Copyright Law is authorized by SPIE subject to payment of copying fees. The Transactional Reporting Service base fee for this volume is $\$ 18.00$ per article (or portion thereof), which should be paid directly to the Copyright Clearance Center (CCC), 222 Rosewood Drive, Danvers, MA 01923. Payment may also be made electronically through CCC Online at copyright.com. Other copying for republication, resale, advertising or promotion, or any form of systematic or multiple reproduction of any material in this book is prohibited except with permission in writing from the publisher. The CCC fee code is 0277-786X/16/\$18.00.

Printed in the United States of America.

Publication of record for individual papers is online in the SPIE Digital Library.

\section{SPIE. DIGITAL}

Paper Numbering: Proceedings of SPIE follow an e-First publication model. A unique citation identifier (CID) number is assigned to each article at the time of publication. Utilization of CIDs allows articles to be fully citable as soon as they are published online, and connects the same identifier to all online and print versions of the publication. SPIE uses a six-digit CID article numbering system structured as follows:

- The first four digits correspond to the SPIE volume number.

- The last two digits indicate publication order within the volume using a Base 36 numbering system employing both numerals and letters. These two-number sets start with 00, 01, 02, 03, 04, $05,06,07,08,09,0 A, 0 B \ldots$ OZ, followed by 10-1Z, 20-2Z, etc. The CID Number appears on each page of the manuscript. 


\title{
Contents
}

\author{
vii Authors \\ ix Conference Committee
}

\section{SESSION 1 WAVEGUIDE ENGINEERING I}

975005 Low-loss polymer optical waveguides with graded-index perfect circular cores for onboard interconnection [9750-3]

975006 Strip loaded waveguide on lithium niobate thin films [9750-4]

\section{SESSION 2 POLARIZATION MANAGEMENT}

975008 ZnO-diffused lithium niobate waveguide polarization controller [9750-6]

975009 Design of a waveguide with optics axes tilted by $45^{\circ}$ and realized by ion-exchange on glass [9750-7]

$9750 \mathrm{OB} \quad$ Birefringence measurement of glass ion-exchanged waveguides: burying depth or cover layer influence [9750-9]

\section{SESSION 3 PLASMONICS}

9750 OD Hybrid-plasmonic three-terminal travelling-wave modulator with dynamic reconfigurability [9750-11]

$9750 \mathrm{OE}$ Integrated angular tracking and plasmonic membrane surfaces for a point of a care refractive index sensor [9750-12]

9750 OF Near infrared plasmonic sensor based on Fano resonance [9750-13]

9750 OG Unidirectional reflectionless propagation in plasmonic waveguide-cavity devices [9750-14]

SESSION 4 WAVEGUIDE ENGINEERING II

$9750 \mathrm{OH} \quad$ Microwave and RF applications for micro-resonator based frequency combs (Invited Paper) [9750-15]

$9750 \mathrm{0J}$ Functionalization of UV-curing adhesives for surface-integrated micro-polymer optical fibers [9750-17] 
9750 OK Integrated-optic tunable chromatic dispersion compensator composed of lattice-form circuit with interleave filter [9750-52]

SESSION 5 ON-CHIP ACTIVE DEVICES

9750 ON Controllable red and blue bandgap energy shifted LEDs and modulators on InGaAsP quantum well platform [9750-21]

975000 Realization of back-side heterogeneous hybrid III-V/Si DBR lasers for silicon photonics [9750-22]

9750 OP Enhancement of the photoluminescence in $\mathrm{Er}$-doped $\mathrm{Al}_{2} \mathrm{O}_{3}$ fabricated by atomic layer deposition [9750-23]

$97500 Q \quad$ Developing the OEIC solutions using two section light-emitting transistor [9750-37]

\section{SESSION 6 ON-CHIP QUANTUM OPTICS}

9750 OT Travelling-wave single-photon detectors integrated with diamond photonic circuits: operation at visible and telecom wavelengths with a timing jitter down to 23 ps [9750-27]

9750 OU Engineering reconfigurable laser-written circuits for practical quantum metrology [9750-28]

\section{SESSION 7 MAGNETO-OPTICS}

9750 OV Faraday polarisation mode conversion in semiconductor waveguides incorporating periodic garnet claddings (Invited Paper) [9750-29]

9750 OW Monolithic on-chip nonreciprocal photonics based on magneto-optical thin films (Invited Paper) [9750-30]

9750 OX Nano-composite magnetic material embedded on $\mathrm{TiO}_{2}$ pillars to realize magneto-optical resonant guided mode gratings [9750-31]

\section{SESSION $8 \quad$ PHOTONIC INTEGRATION}

$97500 Z$ Compact two-mode (de)multiplexer based on MMI couplers with different core thickness on InP [9750-33]

975011 Packaged integrated opto-fluidic solution for harmful fluid analysis [9750-64]

975012 Design and fabrication of adiabatic vertical couplers for hybrid integration by flip-chip bonding [9750-35] 
975014 Integrated optofluidic label-free biosensors using a silicon-nitride-based coupledresonator optical waveguide (Invited Paper) [9750-38]

\section{SESSION 10 MODELLING}

975018 Wave interaction in photonic integrated circuits: Hybrid analytical / numerical coupled mode modeling (Invited Paper) [9750-42]

975019 Leaky waveguides for low к-measurement: From structure design to loss evaluation [9750-43]

$97501 \mathrm{~A} \quad$ Modeling graphene based surface plasmon waveguides and devices [9750-44]

$97501 \mathrm{~B} \quad$ Oblique incidence of semi-guided waves on step-like folds in planar dielectric slabs: Lossless vertical interconnects in 3D integrated photonic circuits [9750-45]

9750 1D Label-free and fluorescence biosensing platform using one dimensional photonic crystal chips (Invited Paper) [9750-47]

$97501 \mathrm{E} \quad$ Fabrication of scattering source for an optical fiber sensor using femtosecond laser internal processing [9750-48]

$97501 \mathrm{H} \quad$ Whispering gallery modes in self-assembled bottle microresonators coupled to planar waveguide [9750-51]

\section{POSTER SESSION}

$97501 \mathrm{~K} \quad$ High-precision opto-mechanical lens system for space applications assembled by innovative local soldering technique [9750-54]

$97501 \mathrm{~L} \quad$ An LSPR fiber optic sensor based on in-line micro-holes fabricated by a second harmonic 400nm femtosecond laser [9750-55]

$97501 \mathrm{P} \quad$ Design of a silicon polarization grating with a sub-wavelength anisotropic structure [9750-59]

9750 IR A novel high sensitive laser diode sensor based on micro-cavity [9750-61]

9750 1T A compact and high-speed plasmonic slot waveguide coupled with photonic waveguide based 2x2 electro-optic switch [9750-63]

$97501 \mathrm{U} \quad$ Ferrofluid-based optical fiber magnetic field sensor fabricated by femtosecond laser irradiation [9750-65] 
$97501 \mathrm{~V}$ Integrated all-polymer Mach-Zehnder interferometers without interaction window in asymmetric configuration [9750-66]

9750 IW Enhancing the resonance stability of a high-Q micro/nanoresonator by an optical means [9750-68] 


\section{Authors}

Numbers in the index correspond to the last two digits of the six-digit citation identifier (CID) article numbering system used in Proceedings of SPIE. The first four digits reflect the volume number. Base 36 numbering is employed for the last two digits and indicates the order of articles within the volume. Numbers start with 00, 01, 02, 03, 04, 05, 06, 07, 08, 09, OA, OB...0Z, followed by 10-1Z, 20-2Z, etc.

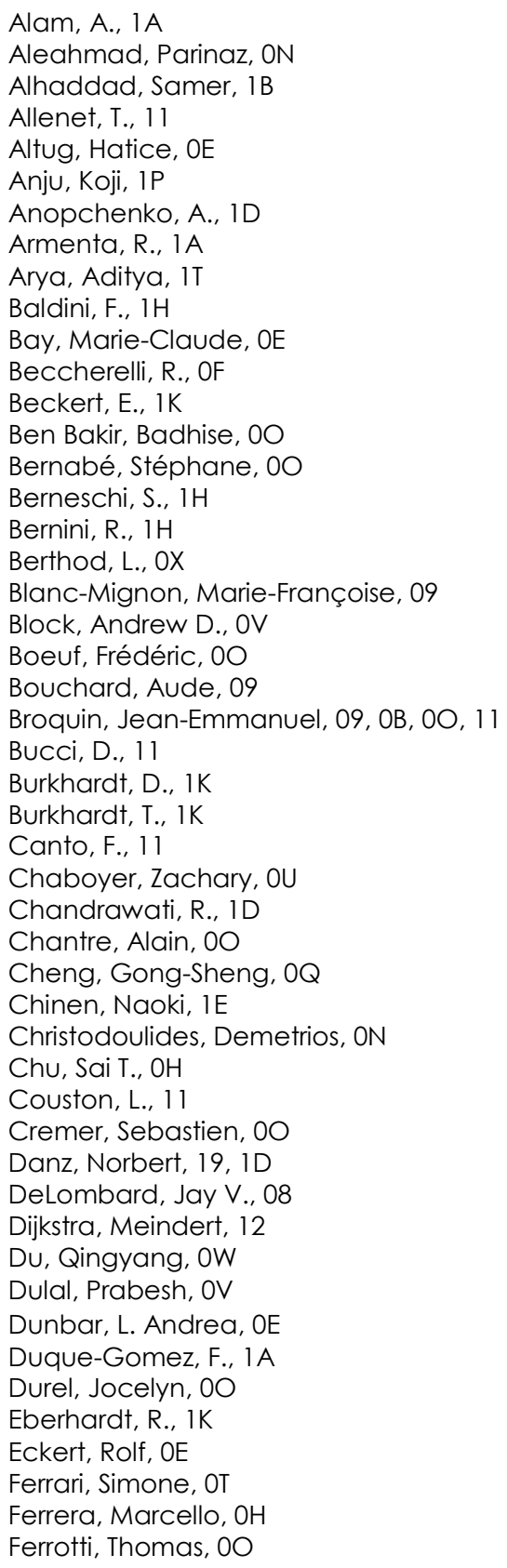

Fiault, G., 1K

Förstner, Jens, 1B

Franzi, Edo, OE

Frydman, C., 1D

Fukagata, Koji, 05

Gamet, E., OX

Garayt, J. P., OB

García-Blanco, Sonia M., 12

Geoffray, F., 11

Ghibaudo, Elise, 09, OB

Goltsman, Gregory N., OT

Guo, Fei, OZ

Goya, Kenji, 1E, $1 \mathrm{~L}$

Gramens, S., 1K

Grenet, Eric, OE

Grimaldi, I. A., 1H

Hachicha, B., OJ

Hammer, Manfred, 18, 1B

Harduin, Julie, 00

Häyrinen, Markus, 06

Heinzelmann, Harry, OE

Helmy, Amr S., OD

Hibti, F.-E., 1D

Hildebrandt, Andre, 1B

Hofmann, Meike, IV

Holmes, Barry M., OV

Honkanen, Seppo, 06

Hornaff, M., 1K

Hsu, Yuan-Fu, OQ

$\mathrm{Hu}$, Juejun, OW

Hua, Liwei, $1 \mathrm{U}$

Huang, Jie, $1 U$

Huang, Y., OG

Hutchings, David C., OV

Ishigure, Takaaki, 05

Jamon, Damien, 09, OB, OX

Jardinier, E., 11

$\mathrm{Ji}$, Chen, $\mathrm{OZ}$

Jordan, Elodie, 09, OB

Kahl, Oliver, OT

Kamm, A, $1 \mathrm{~K}$

Karvinen, Petri, 06

Karvonen, Lasse, OP

Kim, Chil-Min, $1 R$

Kim, Hong-Seung, IR

Kim, Sung-Bock, $1 R$

Koechlin, C., $1 \mathrm{~K}$

Kopp, Christophe, 00

Korneev, Alexander, ОT 
Kovalyuk, Vadim, OT

Kuittinen, Markku, 06

Lei, Jincheng, $1 \mathrm{U}$

Li, Pei, IV

Liang, Shan-Fong, $0 Q$

LiKamWa, Patrick L., ON

Lin, Charles, OD

Lin, Qiang, IW

Lipsanen, Harri, OP

Little, Brent E., OH

Lu, Dan, OZ

Luo, Rui, IW

Maillart, E., 1D

Manivasakan, R., IT

McGuire, D., 1A

Michelotti, Francesco, 19, 1D

Min, C., OG

Mitchell, Arnan, $\mathrm{OH}$

Morandotti, Roberto, $\mathrm{OH}$

Moss, David J., $\mathrm{OH}$

Mu, Jinfeng, 12

Munzert, Peter, 19, 1D

Napione, L., ID

Nebel, Christoph, OT

Neveu, S., OB, OX

Nguyen, Thach G., $\mathrm{OH}$

Niegemann, J., 1A

Nunzi Conti, G., $1 \mathrm{H}$

Occhicone, A., ID

Oh, Kwang-Ryong, 1 R

Onbasli, Mehmet, OW

Overmeyer, L., OJ

Ozdemir, Cenk I., OE

Park, Jung-Min, IR

Parsy, F., OB

Pasquazi, Alessia, $\mathrm{OH}$

Peccianti, Marco, $\mathrm{OH}$

Pernice, Wolfram H. P., OT

Peyghambarian, Nasser, OP

Pollick, Andrea, 08

Pond, J., $1 \mathrm{~A}$

Poon, Andrew W., 14

Pyymäki-Perros, Alexander, OP

Rana, S., 1D

Rath, Patrik, OT

Reid, A., 1 A

Retz, Jason, 08

Reynaud, S., OX

Ribes, P., $1 \mathrm{~K}$

Rizzo, Riccardo, 19

Rönn, John, OP

Ross, Caroline A., OW

Roussey, Matthieu, 06

Royer, François, 09, OB, OX

Saito, Yuki, 05

Säynätjoki, Antti, OP

Schmieder, S., 1D

Sefunc, Mustafa A., 12

Seki, Atsushi, 1E, 1L
Shahada, L., OF

Shavdina, O., OX

Sherif, S. M., OF

Sherman, Stanislav, IV

Shiraishi, Masahiko, 1E, 1L

Shoeiby, Mehrdad, $\mathrm{OH}$

Sinibaldi, A., 1D

Song, Yang, $1 U$

Sonntag, F., 1D

Sriram, Sri, 08

Stadler, Bethanie J. H., OV

Stanley, Ross P., OE

Steel, M. J., OU

Stenger, Vincent E., 08

Stevens, M. M., 1D

Stokes, Alex, OU

Sun, Xuan, IW

Sun, Xue Yin, OW

Sun, Zhipei, OP

Swillam, M., OF

Tabbakh, Thamer, ON

Takiguchi, Koichi, OK

Testa, G., 1H

Toney, James E., 08

Tsuda, Hiroyuki, IP

Tünnermann, A., 1K

Uetsuka, Hisato, IP

Varghese, B., OX

Veillas, C., OX

Veronis, G., OG

Verrier, I., OX

Vetter, Andreas, OT

Wächter, Christoph, 19

Wang, Huitao, $\mathrm{OZ}$

Wang, Jiawei, 14

Wang, Wei, OZ

Wang, Ziyu, IV

Watanabe, Kazuhiro, 1E, $1 \mathrm{~L}$

Withford, Michael J., OU

Wu, Chao-Hsin, OQ

Xiao, Hai, $1 \mathrm{U}$

Xiao, Yanfen, $1 \mathrm{~V}$

$\mathrm{Xu}$, Bojian, 12

Yao, Zhanshi, 14

Yuan, Lei, $1 \mathrm{U}$

Zappe, Hans, $1 \mathrm{~V}$

Zhang, Cui, OV

Zhang, Qi, IU

Zhang, Ruikang, $\mathrm{OZ}$

Zhang, Xi-Cheng, IW

Zografopoulos, D. C., OF 


\section{Conference Committee}

Symposium Chairs

Jean-Emmanuel Broquin, IMEP-LAHC (France)

Shibin Jiang, AdValue Photonics, Inc. (United States)

Symposium Co-chairs

David L. Andrews, University of East Anglia (United Kingdom)

Alexei L. Glebov, OptiGrate Corporation (United States)

Program Track Chair

Yakov Sidorin, Quarles \& Brady LLP (United States)

Conference Chairs

Jean-Emmanuel Broquin, IMEP-LAHC (France)

Gualtiero Nunzi Conti, Istituto di Fisica Applicata Nello Carrara (Italy)

Conference Co-chairs

Christoph M. Greiner, LightSmyth Technologies, Inc. (United States)

Sonia M. García-Blanco, Universiteit Twente (Netherlands)

Conference Program Committee

Pierre Berini, University of Ottawa (Canada)

Romeo Bernini, Istituto per il Rilevamento Elettromagnetico dell'Ambiente (Italy)

Pavel Cheben, National Research Council Canada (Canada)

Xudong Fan, University of Michigan (United States)

Robert A. Norwood, College of Optical Sciences, The University of Arizona (United States)

Min-Cheol Oh, Pusan National University (Korea, Republic of)

François Royer, Université Jean Monnet Saint-Etienne (France)

Jens H. Schmid, National Research Council Canada (Canada)

Yakov Sidorin, Quarles \& Brady LLP (United States)

Christoph A. Wächter, Fraunhofer-Institut für Angewandte Optik und Feinmechanik (Germany)

Session Chairs

1 Waveguide Engineering I

Jean-Emmanuel Broquin, IMEP-LAHC (France) 
2 Polarization Management

Pavel Cheben, National Research Council Canada (Canada)

3 Plasmonics

Jean-Emmanuel Broquin, IMEP-LAHC (France)

4 Waveguide Engineering II

Yakov Sidorin, Quarles \& Brady LLP (United States)

5 On-Chip Active Devices

Sonia M. García-Blanco, Universiteit Twente (Netherlands)

6 On-Chip Quantum Optics

Jean-Emmanuel Broquin, IMEP-LAHC (France)

7 Magneto-Optics

François Royer, Université Jean Monnet Saint-Etienne (France)

8 Photonic Integration

Sonia M. García-Blanco, Universiteit Twente (Netherlands)

9 Optofluidics

Silvia Soria Huguet, Istituto di Fisica Applicata "Nello Carrara" (Italy)

10 Modelling

Christoph A. Wächter, Fraunhofer-Institut für Angewandte Optik und Feinmechanik (Germany)

11 Sensors

Gualtiero Nunzi Conti, Istituto di Fisica Applicata "Nello Carrara" (Italy) 